\title{
Perioperative predictors of delirium and incidence factors in adult patients post cardiac surgery
}

This article was published in the following Dove Press journal: Pragmatic and Observational Research

\author{
Stavros Theologou' \\ Konstantinos \\ Giakoumidakis \\ Christos Charitos ${ }^{2}$ \\ 'Cardiac Surgery Intensive Care Unit, \\ "Evangelismos" General Hospital of \\ Athens, Athens, Greece; ${ }^{2}$ Department \\ of Cardiothoracic Surgery, \\ "Evangelismos" General Hospital of \\ Athens, Athens, Greece
}

Correspondence: Stavros Theologou Cardiac Surgery Intensive Care Unit, "Evangelismos" General Hospital of Athens, 45-47 Ipsilantou Street, 106 76, Athens, Greece

Tel +306972006052

Email cruxtheol@gmail.com
Background: Delirium is a quite common complication in adult patients post-cardiac surgery. The purpose of our study was to identify perioperative characteristics and also focus on incidence factors that could predict delirium in the cardiac surgery intensive care unit (CICU) postoperatively.

Methods: We conducted a prospective study of 179 consecutive patients, who underwent open-heart surgical operation and were admitted to the CICU of a general tertiary hospital in Athens, Greece. The patients were screened for delirium by using the diagnostic tools of Richmond Agitation Sedation Scale (RASS score) and the Confusion Assessment Method - ICU (CAM-ICU). The delirium assessment was carried out on the 1st and the 2 nd postoperative day, and was conducted twice every nursing shift. A short questionnaire on sociodemographics and clinical patient characteristics was used for data collection purposes.

Results: A total of 179 patients who underwent open-heart surgical operation with cardiopulmonary bypass (CPB) were enrolled in our study. The 2-day incidence of postoperative delirium in ICU was $11.2 \%(n=20 / 179)$. The main independent predictors of delirium on the 2 nd postoperative day were neutrophil-to-lymphocyte ratio $(p=0.001)$ and urea levels $(p=0.016)$. Additionally, increased perioperative creatinine $(p=0.006)$ and sodium $(p=0.039)$ levels were significantly associated with delirium occurrence. Furthermore, elevated EuroSCORE ( $p=0.001)$, extended length of stay (LOS) in ICU $(p<0.001)$, and extended LOS with endotracheal tube $(p=0.001)$ were also statistically significant indicators.

Conclusion: Patients with extended LOS with endotracheal tube and prolonged stay in ICU in accordance with peaked urea, neutrophil-to-lymphocyte ratio, creatinine, and sodium levels seem to have a significantly greater probability of developing delirium in the ICU. Further research is needed in the field of postoperative cardiac patients in order to determine the causality and etiology of certain risk factors for delirium.

Keywords: early recognition of delirium, delirium biomarkers, intubation length of stay

\section{Introduction}

Delirium, as defined by the Diagnostic and Statistic Manual 5th edition, is characterized as the fluctuation of changes in the level of consciousness affecting attention, awareness, and cognition of a patient. ${ }^{1}$ This fluctuation finally results in development of delirium. The onset of delirium ranges from a few minutes since discontinuation of sedation to a few days after the patient has been admitted to the intensive care unit (ICU).

Moreover, early recognition of delirium by all staff who deal with the patient has been demonstrated to be of utmost importance for patients' in-hospital progress, as shown in Eden and Foreman's case study. ${ }^{26}$ It is widely documented that a lack of 
recognition and ineffective communication between health professionals play a significant role toward delirium in ICU patients. $^{2-4}$ The main purpose of this study was to focus on this particular subject in cardiac surgery patients and shed light on the process of early identification of the perioperative predictors which would predispose patients to delirium. We also focused on the early recognition of the development of delirium in ICU patients, and our purpose was to illustrate those specific perioperative characteristics that lead to delirium and evaluate the incidence of delirium in cardiac surgery ICU (CICU).

\section{Patients and methods Study design}

The patients included in the study were any elective and nonelective cases who underwent open-heart surgical operations with total cardiopulmonary bypass (CPB).

Three hundred and twenty-seven postoperative cardiac patients with CPB were screened since admission to CICU, and a total of 179 patients were enrolled in the study, which was performed from May until December 2015.

Exclusion criteria were inadequate knowledge of Greek language, hearing or speech impairment, central nervous system cognitive or mental dysfunction prior to operation, drug or alcohol abuse, age $<18$ years, and any patients resuscitated from respiratory or cardiac arrest.

\section{Data collection and delirium screening}

The patients were screened for delirium by using the diagnostic tool of Confusion Assessment Method, ${ }^{5,6}$ for ICU (CAM-ICU). A short questionnaire on sociodemographics and clinical patient characteristics was used for data collection purposes. Patients' medical files were also reviewed, in order to achieve a more analytical view of their medical condition during the study period.

The institutional protocols for anesthesia and ICU sedation were performed with no specific adjustments for the participants in the study. Etomidate and propofol were used for induction in general anesthesia. Sevoflurane was used for maintenance of sedation intraoperatively and, in terms of analgesia, fentalyl was also administered during the operation. ICU sedation was maintained with propofol. Morphine and paracetamol were prescribed for pain relief. All patients were weaned off sedation and extubated once they became cardiovascularly and respiratorily stable with acid/base balance within a normal range.

Delirium assessment was carried out by 2 of the researchers and took place during the 1 st and 2 nd postoperative day.
The assessment was conducted twice in every shift, in order to record and document any alterations or fluctuations in the mental state of the patients.

First, regarding level of sedation, an assessment took place using the diagnostic tool Richmond Assessment Sedation Scale (RASS score). ${ }^{7}$ This is a diagnostic tool enabling health professionals to assess the level of sedation or agitation in a patient by the bedside after the infusion of sedative drugs was discontinued.

The patients would have a positive score (anything below -3 ) and would be defined as comatose if they responded to painful/physical stimulations but would not open their eyes (RASS score -4). If they did not respond to physical/verbal stimuli, they were characterized as having a RASS score equal to -5 .

Should the patient present with a negative score $(>-3$ up to +4 ), they could then be screened for delirium with the diagnostic tool of CAM-ICU.

The CAM-ICU form was used in its Greek validated form, and consent for its clinical use was obtained by the authors. ${ }^{6}$

The CAM aims to document 4 specific traits of delirium: 1) acute alterations in mental status within a certain period of time, 2) inability of the patient to focus attention, 3) altered level of consciousness, and 4) disorganized structure of thinking. Should the patient present features either of 1) and 2) or 3) and 4), the delirium assessment score would then be defined as positive.

In other words, a patient would be defined as delirious if they responded to verbal stimuli by opening their eyes (having a RASS score ranging from -3 up to +4 , which mirrors the level of consciousness) and also presenting (on top of that) with a positive CAM-ICU score.

Delirium was prevented with early mobilization of patients and chest physiotherapy as well as breathing exercises twice a day. In case of delirium onset, nursing interventions were performed in order to alleviate the effects of this condition and haloperidol was also prescribed, titrated to the lowest effective dose as recommended by the American Psychiatric Association. ${ }^{8}$

\section{Statistical analysis}

Categorical and continuous variables are presented as absolute (n) and relative (\%) frequencies and mean (standard deviation) or median (range), respectively. The normality assumption was evaluated using the Kolmogorov-Smirnov criterion ( $p>0.05$ for all variables), histograms, and normal probability plots. Bivariate analyses were conducted and 
included Pearson's $\chi^{2}$ test and $\chi^{2}$ test for trend to determine associations between categorical variables and Student's $t$-test, analysis of variance, Mann-Whitney test and KruskalWallis test to investigate group differences within continuous variables. Correlation between continuous variables was assessed with Pearson's correlation coefficient, while Spearman's correlation coefficient was used to investigate the relationship between a continuous and a categorical variable.

Also, multivariate linear and logistic regressions were performed; the results of the linear regressions are presented by using the coefficients' $\beta$, the $95 \%$ confidence intervals, and the corresponding $p$-values, while the results of the logistic regressions are presented by using the odds ratios, the $95 \%$ confidence intervals, and the corresponding $p$-values.

A 2-sided $p$-value of 0.05 was considered statistically significant. The Statistical Package for Social Sciences (IBM Corporation, Armonk, NY, USA) program, version 20.0, was used for statistical analysis.

\section{Ethics}

The data collection was conducted after permission was issued by the Scientific Board of "Evangelismos" General Hospital prior to the start of the study.

The participants of the study gave their written informed consent preoperatively to be included in the study, so that they would be assessed for delirium in ICU. The investigation was carried out in accordance with the ethical standards of the responsible institutional committee for human experimentation and following the tenets of the Helsinki Declaration of 1975, as revised in 2013. Precautionary measures were taken in order to protect the privacy of the patients and the confidentiality of their personal data, in order to use the minimum amount of information necessitated throughout the study.

\section{Results}

Sociodemographic and perioperative data and significant biomarker findings

The mean age of patients in this study was 63.3 years, and the majority of them were males (72.1\%). Approximately half of the patients underwent exclusively coronary artery bypass grafting (CABG) $(52.5 \%)$, and nearly a quarter of all patients underwent aortic (AVR) or mitral valve replacement (MVR) (25.1\%). In Table 1, we present a summary of the sociodemographic, clinical, and perioperative patients' characteristics. The mean ICU and in-hospital length of stay (LOS) were 1.6 and 7.4 days, respectively. In addition, 21 patients $(11.7 \%)$ were transferred to the high-dependency
Table I Demographic, clinical, and perioperative patients' characteristics

\begin{tabular}{|c|c|}
\hline Characteristics & Mean ( \pm SD) \\
\hline Age (years) & $63.3(12.7)$ \\
\hline BMI $\left(\mathrm{kg} / \mathrm{m}^{2}\right)$ & $28.1(5.1)$ \\
\hline CPB (min) & $121.2(42.6)$ \\
\hline Ischemia time during CPB (min) & $77.4(31.5)$ \\
\hline ICU LOS (hours) & $35.1(19.8)$ \\
\hline ICU LOS (days) & $1.6(1.8)$ \\
\hline In-hospital LOS (days) & $7.4(2.9)$ \\
\hline Total sedation time (hours) & $7.2(8.4)$ \\
\hline LOS with endotracheal tube (hours) & $10.7(11.4)$ \\
\hline \multirow[t]{2}{*}{ EuroSCORE II (\%) } & $4(6.0)$ \\
\hline & n (\%) \\
\hline \multicolumn{2}{|l|}{ Gender } \\
\hline Male & $129(72.1)$ \\
\hline Female & $50(27.9)$ \\
\hline \multicolumn{2}{|l|}{ Type of surgical operation } \\
\hline CABG & $94(52.5)$ \\
\hline AVR/MVR & $45(25.1)$ \\
\hline Bentall/-ascending aorta $( \pm$ arch $)$ replacement & $12(6.7)$ \\
\hline$A V R+M V R( \pm C A B G)$ & $23(12.8)$ \\
\hline ASD/VSD & $5(2.8)$ \\
\hline \multicolumn{2}{|l|}{ Transfer to HDU } \\
\hline Yes & $21(11.7)$ \\
\hline No & $158(88.3)$ \\
\hline \multicolumn{2}{|l|}{ Blood transfusion } \\
\hline Yes & $13(7.3)$ \\
\hline No & $166(92.7)$ \\
\hline
\end{tabular}

Abbreviations: AVR, aortic valve replacement; $B M I$, body mass index; CABG, coronary artery bypass grafting; CPB, cardiopulmonary bypass; HDU, highdependency unit; ICU, intensive care unit; LOS, length of stay; MVR, mitral valve replacement.

unit (HDU) after ICU discharge as they were not completely safe to be transferred to the ward yet.

Thirteen out of 179 patients (7.3\%) were given blood transfusion, and we also recorded a mean sedation time of 7.2 hours and a mean time of intubation at 10.7 hours. As our unit has a form of cardiac surgery recovery department, we also measured the mean time of ICU LOS in hours (35.1 hours).

Delirium was recorded in 20 out of 179 patients in this study (Table 2). The 2-day incidence of delirium in ICU was $11.2 \%(n=20 / 179)$.

We conducted bivariate associations between demographic, perioperative, and patients' data and the outcome of delirium.

In Table 3, looking into the types of surgical operation, delirium was recorded more frequently in CABG cases ( 6 out of 20, $p=0.032$ ) and AVR + MVR ( \pm CABG) cases (10 out of 20, $p<0.001$ ). An elevated EuroSCORE II (mean 6.3) was more significantly correlated with delirium $(p=0.001)$. 
Table 2 Patient outcomes

\begin{tabular}{ll}
\hline Outcome & $\mathbf{N}(\%)$ \\
\hline Delirium & \\
No & $159(88.8)$ \\
Yes & $20(11.2)$ \\
\hline
\end{tabular}

Table 3 Types of surgical operation and perioperative patient characteristics and outcomes associated with delirium

\begin{tabular}{|c|c|c|c|c|}
\hline \multirow{2}{*}{$\begin{array}{l}\text { Surgical } \\
\text { operation }\end{array}$} & \multirow{2}{*}{$\begin{array}{l}\text { Number of } \\
\text { patients, } \mathbf{N}(\%)\end{array}$} & \multicolumn{2}{|c|}{ Outcome (delirium) } & \multirow[t]{2}{*}{$p$-value } \\
\hline & & No & Yes & \\
\hline \multicolumn{4}{|l|}{$\overline{\mathrm{CABG}}$} & $0.032^{\mathrm{a}}$ \\
\hline No & & $71(44.7)$ & $14(70.0)$ & \\
\hline Yes & $94(52.5)$ & $88(55.3)$ & $6(30.0)$ & \\
\hline \multicolumn{4}{|l|}{ AVR/MVR } & $0.267^{\mathrm{a}}$ \\
\hline No & & $117(73.6)$ & $17(85.0)$ & \\
\hline Yes & $45(25.1)$ & $42(26.4)$ & $3(15.0)$ & \\
\hline \multicolumn{4}{|c|}{ Bentall/_tascending aorta ( \pm arch) replacement } & $0.746^{\mathrm{a}}$ \\
\hline No & & $148(93.1)$ & $19(95.0)$ & \\
\hline Yes & $12(6.7)$ & II (6.9) & I (5.0) & \\
\hline \multicolumn{4}{|c|}{$A V R+M V R( \pm C A B G)$} & $<0.00 I^{\mathrm{a}}$ \\
\hline No & & | 46 (9l.8) & $10(50.0)$ & \\
\hline Yes & $23(12.8)$ & $13(8.2)$ & $10(50.0)$ & \\
\hline \multicolumn{4}{|l|}{ ASD/VSD } & $0.549^{\mathrm{a}}$ \\
\hline No & & 154 (96.9) & $20(100.0)$ & \\
\hline Yes & $5(2.8)$ & $5(3.1)$ & $0(0.0)$ & \\
\hline \multicolumn{4}{|c|}{ Transfer to HDU } & $0.321^{c}$ \\
\hline No & I58 (88.3) & $139(87.4)$ & $19(95.0)$ & \\
\hline Yes & $21(11.7)$ & $20(12.6)$ & I (5.0) & \\
\hline \multicolumn{4}{|c|}{ Blood transfusion } & $0.157^{\mathrm{a}}$ \\
\hline No & $166(92.7)$ & 149 (93.7) & $17(85.0)$ & \\
\hline Yes & $13(7.3)$ & $10(6.3)$ & $3(15.0)$ & \\
\hline \multirow[t]{2}{*}{ EuroSCORE Il $\mathrm{l}^{\mathrm{b}, \mathrm{c}}$} & $6.2(8.9) /$ & I.7 (2/6)/ & $3.7(5.3) /$ & $0.00 \mathrm{I}^{\mathrm{d}}$ \\
\hline & I.I (I3.7) & $3.7(5.9)$ & $6.3(6.6)$ & \\
\hline
\end{tabular}

Notes: Values are presented as $n(\%)$ unless they are mentioned otherwise. ${ }^{2} \chi^{2}$ test, ${ }^{b}$ median (interquartile range), ' mean (standard deviation), ${ }^{\mathrm{d}}$ Mann-Whitney test. Bold data are statistically significant.

Abbreviations: ASD, atrial septal defect; AVR, aortic valve replacement; CABG, coronary artery bypass grafting; HDU, high-dependency unit; ICU, intensive care unit; MVR, mitral valve replacement; VSD, ventricular septal defect.
Looking into patient-related outcomes in Table 4, we noticed the following findings: mean and median time of ICU LOS (days) for patients with delirium were higher compared to mean and median time in patients with no delirium $(p<0.001)$.Mean and median time of in-hospital LOS (days) for patients with delirium were higher compared to mean and median time of patients with no delirium $(p=0.039)$.

Mean and median time of ICU LOS (h) for patients with delirium was higher compared to mean time of patients with no delirium $(p<0.001)$.

Median and mean time of LOS with endotracheal tube for patients with delirium were both higher compared to median and mean time of patients with no delirium $(p=0.001)$.

In Table 5, the results showed that patients who developed delirium postoperatively were significantly older (mean age: 69 vs 62 years, $p=0.020$ ). They also had higher body mass index (29.2 vs $27.9, p=0.226$ ), but without statistical significance.

Regarding the operation procedure data, total $\mathrm{CPB}$ and total ischemia time during $\mathrm{CPB}$ were both prolonged in patients with delirium (140.2 vs 118.8 minutes, $p$-value $=0.113$ and 90.1 vs 75.8 minutes, $p$-value $=0.155$, respectively) but were not statistically significant.

Regarding ICU conditions, patients with delirium remained sedated and intubated for longer periods of time (6.2 vs 5 hours, $p=0.189$ and 11.5 vs 8 hours, $p=0.001$, respectively).

In terms of total ICU LOS, patients with delirium stayed in the unit for a longer period, and this was statistically significant (45.5 vs 26 hours, $p<0.001)$.

In terms of in-hospital LOS, the difference was not clinically significant, but it was found to be statistically significant; patients with delirium were hospitalized for 7.5 days, while patients without delirium stayed for 7 days $(p=0.028)$.

Table 4 Bivariate associations between delirium and patient perioperative outcomes

\begin{tabular}{|c|c|c|c|c|c|}
\hline & \multirow{2}{*}{$\begin{array}{l}\text { Mean (standard } \\
\text { deviation) }\end{array}$} & \multirow{2}{*}{$\begin{array}{l}\text { Median (interq } \\
\text { range) }\end{array}$} & \multicolumn{2}{|l|}{ Outcome delirium } & \multirow[t]{2}{*}{$p$-value } \\
\hline & & & No & Yes & \\
\hline CPB (min) & $121.2(42.6)$ & $42.6(1 \mid 3.0)$ & II $8.8(40.2)$ & $140.2(56.0)$ & $0.113^{\mathrm{a}}$ \\
\hline Ischemia time during CPB (min) & $77.4(31.5)$ & $31.5(70.0)$ & $75.8(29.7)$ & $90.1(42.0)$ & $0.155^{\mathrm{a}}$ \\
\hline ICU LOS (days) $)^{b, c}$ & $1.6(1.8)$ & $1.8(1.0)$ & $1.5(0.7) / 1.0(1.0)$ & $2.4(1.6) / 2.0(1.8)$ & $<0.00 I^{d}$ \\
\hline In-hospital LOS (days) & $7.4(2.9)$ & $2.9(7.0)$ & $7.3(2.7) / 7.0(2.0)$ & $8.8(4.6) / 7.5(3.5)$ & $0.028^{d}$ \\
\hline ICU LOS (h) & $35.1(19.8)$ & $19.8(29.0)$ & $32.6(14.5) / 26.0(19.0)$ & $55.9(37.7) / 45.5(31.2)$ & $<0.001^{d}$ \\
\hline Total sedation time $(h)^{\mathrm{b}, \mathrm{c}}$ & $7.2(8.4)$ & $8.4(5.0)$ & $6.5(5.0) / 5.0(3.0)$ & 13.2(20.7)/6.3 (6.6) & $0.189^{d}$ \\
\hline Length of stay with endotracheal tube $(h)^{\mathrm{b}, \mathrm{c}}$ & $10.7(11.4)$ & I I.4 (8.0) & $9.7(8.1) / 8.0(4.0)$ & $19.5(24.1) / 1 \mid 1.5(10.6)$ & $0.00 I^{d}$ \\
\hline
\end{tabular}

Notes: Values are presented as mean (standard deviation) unless they are mentioned otherwise. ${ }^{\mathrm{a}} \mathrm{t}$-test, ${ }^{\mathrm{b}}$ mean (standard deviation), ${ }^{\mathrm{c}}$ median (interquartile range), ${ }^{\mathrm{d}}$ MannWhitney test. Bold data are statistically significant.

Abbreviations: CPB, cardiopulmonary bypass; ICU, intensive care unit; LOS, length of stay. 
Table 5 Bivariate associations between demographic and other data and delirium development

\begin{tabular}{|c|c|c|c|}
\hline \multirow[t]{2}{*}{ Independent variable } & \multicolumn{2}{|l|}{ Delirium } & \multirow[t]{2}{*}{$p$-value } \\
\hline & No & Yes & \\
\hline Age $^{a}$ & $62.5(12.6)$ & $69.6(11.9)$ & $0.020^{b}$ \\
\hline Sex & & & $0.202^{c}$ \\
\hline Male & $117(73.6)$ & $12(60.0)$ & \\
\hline Female & $42(26.4)$ & $8(40.0)$ & \\
\hline Weight $^{\mathrm{a}}$ & $81.0(17.0)$ & $81.4(12.2)$ & $0.906^{\mathrm{b}}$ \\
\hline Height $^{\mathrm{a}}$ & $170.1(8.1)$ & $167.2(10.4)$ & $0.230^{\mathrm{b}}$ \\
\hline $\mathrm{BMl}^{\mathrm{a}}$ & $27.9(5.2)$ & $29.2(4.2)$ & $0.226^{\mathrm{b}}$ \\
\hline \multicolumn{4}{|l|}{ Surgical type of operation } \\
\hline CABG & & & $0.032^{c}$ \\
\hline No & 7I (44.7) & $14(70.0)$ & \\
\hline Yes & $88(55.3)$ & $6(30.0)$ & \\
\hline AVR/MVR & & & $0.267^{c}$ \\
\hline No & $117(73.6)$ & $17(85.0)$ & \\
\hline Yes & $42(26.4)$ & $3(15.0)$ & \\
\hline Bentall \pm ascending aorta \pm arch replacement & & & $0.746^{c}$ \\
\hline No & $148(93.1)$ & $19(95.0)$ & \\
\hline Yes & II (6.9) & I (5.0) & \\
\hline AVR and MVR ( \pm CABG) & & & $<0.00 I^{\mathrm{c}}$ \\
\hline No & $146(9 \mid .8)$ & $10(50.0)$ & \\
\hline Yes & $13(8.2)$ & $10(50.0)$ & \\
\hline ASD/VSD & & & $0.549^{c}$ \\
\hline No & $154(96.9)$ & $20(100.0)$ & \\
\hline Yes & $5(3.1)$ & $0(0.0)$ & \\
\hline Transfer to HDU & & & $0.321^{c}$ \\
\hline No & $139(87.4)$ & $19(95.0)$ & \\
\hline Yes & $20(12.6)$ & I (5.0) & \\
\hline EuroSCORE $\|^{\mathrm{a}, \mathrm{d}}$ & $1.7(2.6) / 3.7(5.9)$ & $3.7(5.3) / 6.3(6.6)$ & $0.00 I^{\mathrm{e}}$ \\
\hline $\mathrm{CPB}(\min )^{\mathrm{a}}$ & $118.8(40.2)$ & $140.2(56.0)$ & $0.113^{b}$ \\
\hline Ischemia time during CPB $(\mathrm{min})^{\mathrm{a}}$ & $75.8(29.7)$ & $90.1(42.0)$ & $0.155^{b}$ \\
\hline In-hospital LOS (days) ${ }^{d}$ & $7.0(2.0)$ & $7.5(3.5)$ & $0.028^{f}$ \\
\hline ICU LOS (hours) ${ }^{d}$ & $26.0(19.0)$ & $45.5(31.2)$ & $<0.00 \mathrm{I}^{\mathrm{f}}$ \\
\hline Total sedation time (hours) ${ }^{d}$ & $5.0(3.0)$ & $6.2(6.6)$ & $0.189^{f}$ \\
\hline LOS with endotracheal tube (hours) ${ }^{d}$ & $8.0(4.0)$ & II.5 (I0.6) & $0.00 \mathrm{I}^{\mathrm{f}}$ \\
\hline NL ratio, 2nd postoperative day ${ }^{d}$ & $9.2(7.3)$ & $16.4(17.9)$ & $0.00 \mathrm{I}^{\mathrm{f}}$ \\
\hline Urea operation day ${ }^{d}$ & $41.0(19.0)$ & $49.5(29.0)$ & $0.025^{f}$ \\
\hline Urea 2 nd postoperative day ${ }^{d}$ & $40.0(25.0)$ & $52.5(45.5)$ & $0.016^{f}$ \\
\hline Creatinine operation day ${ }^{d}$ & $\mathrm{I} .0(0.3)$ & I.I (0.5) & $0.018^{f}$ \\
\hline Creatinine Ist postoperative day ${ }^{d}$ & $1.0(0.5)$ & I. $3(0.5)$ & $0.006^{f}$ \\
\hline Creatinine 2 nd postoperative day ${ }^{d}$ & $1.0(0.5)$ & $1.3(0.5)$ & $0.026^{f}$ \\
\hline Sodium Ist postoperative day ${ }^{\mathrm{a}}$ & I $42.7(3.6)$ & I $44.5(3.4)$ & $0.039^{b}$ \\
\hline Sodium 2nd postoperative day ${ }^{a}$ & $141.2(3.7)$ & $143.4(4.0)$ & $0.033^{b}$ \\
\hline PT operation daya & $13.1(1.3)$ & $14.4(2.4)$ & $<0.00 \mathrm{I}^{\mathrm{b}}$ \\
\hline INR operation day ${ }^{a}$ & $1.0(0.1)$ & $\mathrm{I} .2(0.2)$ & $<0.00 I^{b}$ \\
\hline
\end{tabular}

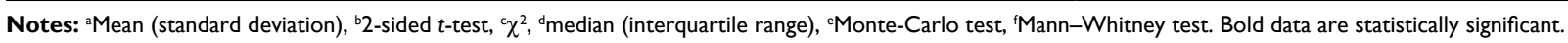
Abbreviations: ASD, atrial septal defect; AVR, aortic valve replacement; BMI, body mass index; CABG, coronary artery bypass grafting; CPB, cardiopulmonary bypass; HDU, high-dependency unit; ICU, intensive care unit; INR, International Normalized Ratio; LOS, length of stay; MVR, mitral valve replacement; NL, neutrophil-to-lymphocyte; PT, prothrombin time; VSD, ventricular septal defect.

Our intention was to emphasize on the importance of certain biomarkers in order to use them in everyday regular assessment for early delirium prognosis.

In that sense, urea and creatinine levels were found to be statistically significant on the day of the operation (patients with and without delirium: 49.5 vs $41, p=0.025$ and 1.1 vs 1 , $p=0.018$, respectively); an increase in creatinine levels was also found to be statistically significant on the first and second postoperative days ( $1 \mathrm{st}$ and 2 nd postoperative day, 1.3 vs $1, p=0.006$ and 1.3 vs $1, p=0.026$ ), and we also found urea levels on the second day to be significantly elevated ( 40 vs $52.5, p=0.016$ ).

In Table 6, we present a full view of the bivariate associations between perioperative blood results and the outcome of delirium in the study. 
Table 6 Bivariate associations between perioperative blood results and delirium

\begin{tabular}{|c|c|c|c|}
\hline \multirow[t]{2}{*}{ Characteristics } & \multirow{2}{*}{$\begin{array}{l}\text { Delirium } \\
\text { No }\end{array}$} & \multirow{2}{*}{$\frac{\text { Delirium }}{\text { Yes }}$} & \multirow[t]{2}{*}{$p$-value } \\
\hline & & & \\
\hline NL ratio, operation day ${ }^{\mathrm{a}}$ & $2.2(1.7) / 3.1(3.0)$ & $2.9(1.9) / 4.6(4.8)$ & $0.06 \mathrm{I}^{\mathrm{b}}$ \\
\hline NL ratio, Ist postoperative day & $14.0(9.1) / 15.2(7.3)$ & $16.0(11.6) / 23.2(22.0)$ & $0.166^{b}$ \\
\hline NL ratio, 2nd postoperative day ${ }^{\mathrm{a}}$ & $9.3(7.1) / 10.8(6.8)$ & $16.4(17,9) / 18.1(10.4)$ & $0.00 I^{b}$ \\
\hline Hematocrit operation day & $38.0(4.8)$ & $36.9(4.4)$ & $0.292^{c}$ \\
\hline Hematocrit Ist postoperative day & $32.5(3.9)$ & $30.4(3.3)$ & $0.013^{c}$ \\
\hline Hematocrit 2nd postoperative day & $30.9(3.6)$ & $28.7(3.0)$ & $0.005^{c}$ \\
\hline Hb operation day & $12.8(1.8)$ & $12.2(1.6)$ & $0.105^{c}$ \\
\hline $\mathrm{Hb}$ Ist postoperative day & $10.9(1.4)$ & $10.0(1.1)$ & $0.004^{c}$ \\
\hline $\mathrm{Hb}$ 2nd postoperative day & $10.3(1.3)$ & $9.4(1.0)$ & $0.00 I^{c}$ \\
\hline Plt operation day & $223.6(65.9)$ & $215.5(62.6)$ & $0.588^{c}$ \\
\hline Plt Ist postoperative day & I88.2 (67.1) & $149.9(52.0)$ & $0.006^{c}$ \\
\hline Plt 2nd postoperative day & $175.6(66.9)$ & I $35.4(54.4)$ & $0.005^{c}$ \\
\hline Urea operation day & $41.0(19.0) / 44.8(22.0)$ & $49.5(29.0) / 53.9(23.1)$ & $0.025^{c}$ \\
\hline Urea Ist postoperative day ${ }^{\mathrm{a}}$ & $40.0(23.0) / 45.5(22.3)$ & $47.5(35.3) / 52.9(26.1)$ & $0.126^{\mathrm{b}}$ \\
\hline Urea 2 nd postoperative day ${ }^{a}$ & $40.0(25.0) / 48.0(26.1)$ & $52.5(45.5) / 62.8(30.5)$ & $0.016^{b}$ \\
\hline Creatinine operation day ${ }^{\mathrm{a}}$ & $1.0(0.3) / 1.2(1.0)$ & I.I (0.6)/I2 (0.4) & $0.018^{b}$ \\
\hline Creatinine Ist postoperative day ${ }^{\mathrm{a}}$ & $10(0.5) / 1.2(1.0)$ & $1.3(0.5) / 1.3(0.4)$ & $0.006^{b}$ \\
\hline Creatinine 2 nd postoperative day ${ }^{a}$ & $1.0(0.5) / 1.2(1.0)$ & $1.3(0.5) / 1.3(0.6)$ & $0.026^{b}$ \\
\hline Sodium operation day & $140.5(3.1)$ & $141.3(4.1)$ & $0.433^{c}$ \\
\hline Sodium Ist postoperative day & $142.7(36)$ & $1445(34)$ & $0.039^{c}$ \\
\hline Sodium 2 nd postoperative day & $14 \mid .2(3.7)$ & $143.4(4.0)$ & $0.033^{c}$ \\
\hline Albumin operation day & $4.0(0.6)$ & $3.9(0.4)$ & $0.136^{c}$ \\
\hline Albumin Ist postoperative day & $3.5(0.4)$ & $3.4(0.4)$ & $0.27 I^{c}$ \\
\hline Albumin 2 nd postoperative day & $3.5(0.4)$ & $3.5(0.4)$ & $0.405^{c}$ \\
\hline CRP operation day & $0.4(1.3) / 1.7(3.3)$ & $0.3(1.6) / 1.1(1.4)$ & $0.719^{b}$ \\
\hline CRP Ist postoperative day & $7.4(7.3) / 8.8(6.6)$ & $5.6(6.4) / 6.9(6.0)$ & $0.183^{b}$ \\
\hline CRP 2nd postoperative day & $16.5(14.1) / 16.6(8.8)$ & $13.2(\mid 1.4) / 13.6(7.9)$ & $0.163^{b}$ \\
\hline PT operation day & $13.1(1.3)$ & $14.4(2.4)$ & $<0.00 I^{c}$ \\
\hline PT Ist postoperative day & I4.I (3.0) & $14.6(2.8)$ & $0.448^{c}$ \\
\hline PT 2nd postoperative day & $14.5(5.6)$ & $16.3(9.0)$ & $0.397^{c}$ \\
\hline INR operation day & $\mathrm{I} .0(0.1)$ & I.2(0.2) & $<0.00 I^{c}$ \\
\hline INR Ist postoperative day & I.I (0.3) & $1.2(0.2)$ & $0.529^{c}$ \\
\hline INR 2nd postoperative day & $1.2(0.4)$ & I.3 $(0.8$ & $0.375^{c}$ \\
\hline
\end{tabular}

Notes: Values are presented as mean (standard deviation) unless they are mentioned otherwise. a Median (interquartile range), ${ }^{b}$ Mann-Whitney test, ${ }^{c}$ paired $t$-test. Bold data are statistically significant.

Abbreviations: CRP, C-reactive protein; Hb, hemoglobin; INR, International Normalized Ratio; NL, neutrophil-to-lymphocyte; Plt, platelet; PT, prothrombin time.

Looking into blood results on the operation day, neutrophil-to-lymphocyte ratio (NL ratio) was found with a $p$-value $=0.061$ (mean 4.6 vs 3.1 ), on the 1 st day was not significant (mean 23.2 vs $15.2, p=0.166$ ) but on the second postoperative day it was recorded as statistically significant (patients with delirium vs non delirium 18.1 vs $10.8, p=0.001$ ).

We also noticed that hematocrit, $\mathrm{Hb}$, and platelets for both the postoperative days of the study were correlated with delirium development.

As for clotting blood results, prolonged prothrombin time (PT) and International Normalized Ratio (INR) on the operation day were correlated with delirium $(p<0.001)$.

Finally, sodium levels were also found to present a level of statistical significance for both days ( $p$-value $=0.039$ and $p$-value $=0.033$, respectively), so this could be correlated with the presence of delirium.
By using multiple logistic regressions, as shown in Table 7, we reached the conclusion that the patients who stayed longer in ICU were more susceptible to develop delirium (odds ratio 1.043 and $p=0.011$ ).

In addition, patients who underwent AVR + MVR \pm CABG were prone to develop delirium more frequently compared to patients who did not have that type of operation (odds ratio 12.157, $p<0.001$ ).

Moreover, the patients who had increased NL ratio levels on the second postoperative day had a higher chance of delirium $(p=0.026)$.

\section{Discussion}

In general, delirium is well noted as a quite common complication in cardiac surgical patients. Li et al ${ }^{9}$ and Bakker et $\mathrm{al},{ }^{10}$ among others, have mentioned that there has been documented 
Table 7 Multivariable logistic regression with delirium as dependent variable

\begin{tabular}{llll}
\hline & $\begin{array}{l}\text { Odds } \\
\text { ratio }\end{array}$ & $\begin{array}{l}\mathbf{9 5 \%} \text { confidence } \\
\text { interval }\end{array}$ & p-value \\
\hline ICU LOS (hours) & 1.043 & $1.010-1.078$ & $\mathbf{0 . 0 1 I}$ \\
NL ratio, 2nd & 1.077 & $1.009-1.151$ & $\mathbf{0 . 0 2 6}$ \\
postoperative day & & & \\
AVR + MVR $( \pm$ CABG) & 12.157 & $3.410-43.338$ & $<0.001$ \\
\hline
\end{tabular}

Note: Bold data are statistically significant.

Abbreviations: AVR, aortic valve replacement; CABG, coronary artery bypass grafting; ICU, intensive care unit; LOS, length of stay; MVR, mitral valve replacement; NL, neutrophil-to-lymphocyte.

a wide variety of cases with patients in delirium (10\%-73\%) postoperatively and large discrepancies have been recorded in documentation, data collection, and etiology regarding the "state of delirium" in the relevant published studies and papers.

In addition, it is well understood that delirium is a challenging clinical condition that becomes costly and affects health services and their staff in many aspects, as stated in the paper by Weinrebe et $\mathrm{al}^{11}$ and Lee and Kim. ${ }^{12}$

Franco et $\mathrm{al}^{13}$ and Weinrebe et al ${ }^{11}$ also state, in their economic evaluation, that the level of provided health services consists of nursing and medical workload point of view, and financial resources' point of view as well.

Androsova et $\mathrm{a} \mathrm{l}^{14}$ and Rudolph et $\mathrm{a} \mathrm{l}^{15}$ make a remarkable point regarding systemic inflammatory response as a major clinical condition postoperatively, which potentially might affect the brain as well.

There needs to be a clear distinction between long-term delirium in critical care patients and short-term postoperative delirium in cardiac patients.

In recent years, it has been well established in literature that 3 distinct types of delirium exist: the hypoactive subtype, the hyperactive subtype, and the mixed motor subtype. It has also been well recognized that the hypoactive form of delirium is the one most susceptible to deterioration in patient condition. ${ }^{27,28}$ Arend and Christensen ${ }^{5}$ and Meagher $^{3}$ and Stransky et $\mathrm{al}^{4}$ in their studies show that a hypoactive patient with delirium is not easily recognized since onset; moreover, complications and side effects may have already taken place, thus directing the patient to a major condition well known as that of a "very sick patient".

Our intention was to emphasize on the importance of certain biomarkers in order to use them in the everyday routine assessment for early delirium prognosis.

This is why we exclusively investigated these biomarkers postoperatively as these could be easily measured and, above all, could render results at a low cost and much more quickly, with no extra cost apart from the everyday routine blood tests.
In that sense, we conducted a bivariate analysis between perioperative patients' characteristics and the occurrence of delirium.

A multiple logistic regression was conducted therefore, with delirium appearance as a dependent covariate. The variables that were introduced in the logistic regression model were the result of the bivariate analysis that preceded the multivariate analysis; the variables that were introduced in the model were those that showed a statistically significant relationship at the level of $0.05(p<0.05)$ with the dependent variable (occurrence of delirium). It is well known that the development of delirium in postsurgical patients is a multifactorial phenomenon. The collection of the added required sample constituted a time-consuming and costly process, and it was not possible to include in the model fewer independent variables nor collect a larger sample during the period of the study.

In terms of perioperative predictors, Norkiene et $\mathrm{al}^{2}$ in 2007 indicated the significance of specific factors which contribute to the appearance of delirium, such as prolonged sedation time, prolonged mechanical ventilation time, and prolonged length of ICU stay.

Our results, as demonstrated in Tables 4 and 5, also show that patients with prolonged ICU and in-hospital LOS developed delirium more frequently compared to patients with shorter LOS.

Norkiene et $\mathrm{al}^{2}$ and Zhang et $\mathrm{al}^{16}$ stated in their studies that patients with prolonged LOS were prone to developing delirium more frequently, and this finding was similar to our study.

Moreover, as presented in Tables 4 and 5, we also noticed that older age, elevated EuroSCORE II, prolonged LOS with endotracheal tube, and prolonged ICU LOS were found to be statistically and clinically significant and were also consistent with similar findings in studies by Shadvar et a $\mathrm{l}^{17}$ and Bakker et al. ${ }^{10}$

Total sedation time was also found prolonged in patients with delirium compared to patients with no delirium but was not statistically significant (6.2 vs 5.0, $p=0.189$ ).

$\mathrm{CPB}$ and ischemia time, as shown in Tables 4 and 5, were significantly prolonged but not statistically associated with delirium, whereas these findings were found increased in the study of Li et al. ${ }^{9}$

We also noticed, as seen in Table 3, that 13 patients out of 179 had a blood transfusion, but this was not correlated with delirium significantly ( $p=0.157)$. On the other hand, regarding the everyday routine blood results, as shown in Table 6, changes in postoperative hematocrit, $\mathrm{Hb}$, and platelet 
numbers seem to raise the alarm for delirium development. A similar point about low $\mathrm{Hb}$ and anemia is also made in Norkiene et al's ${ }^{2}$ study.

In terms of emphasizing the results of certain biomarker tests, Sunbul et al ${ }^{18}$ remind us that neutrophils and leukocytes are of outmost importance for the development of inflammation, and we also have to bear in mind that Giakoumidakis et $\mathrm{al}^{19}$ and Tan et $\mathrm{al},{ }^{20}$ among others, have recently investigated NL ratio as a potential biomarker for systemic inflammatory response syndrome in cardiac patients.

We also have to mention that in Egberts' and MattaceRaso's study ${ }^{21}$ in acutely ill hospitalized patients, higher NL ratio in patients with delirium was found.

Moreover, we noticed that Kulaksizoglu and Kulaksizoglu ${ }^{22}$ in their study investigated the correlation of oxidative stress and inflammation with elevated NL ratio in patients with schizophrenia.

Given all these facts and given that the severity of depression has been associated with NL ratio, ${ }^{20}$ we hypothesized that since NL ratio was "responsible" for systemic inflammatory response syndrome and depression, we could try to investigate if there existed some sort of correlation of this biomarker with delirium as well.

In our study we also noticed that, looking into the blood results given in Table 6 , NL ratio on the operation day had a $p$-value $=0.061$ (mean 4.6 vs 3.1$)$, on the 1 st day it was not significant (mean 23.2 vs $15.2, p=0.166$ ) but on the second postoperative day it was recorded as statistically significant (patients with delirium vs without delirium 18.1 vs $10.8, p=0.001$ ).

Multiple regression analysis, as presented in Table 7 , revealed that patients with elevated NL ratio on the second postoperative day were more susceptible to develop delirium compared to patients with a lower NL ratio, and the $p$-value was measured as 0.026 .

It was also observed that patients undergoing more complicated intraoperative procedures (2-valve replacement) had a higher chance of delirium, as in Norkiene et al's ${ }^{2}$ study.

Urea and creatinine levels, as presented in Tables 5 and 6 , were also found to be increased as there were cases with deteriorated preoperative renal function.

Tsuruta and $\mathrm{Oda}^{25}$ has indicated the predictive role of certain biomarkers such as IL-6 and TNF on the increased risk of delirium development. In several studies, procalcitonine and C-reactive protein levels have also been found to be increased, indicating the association between the onset of delirium and increased levels of these biomarkers both in infectious and noninfectious conditions, as demonstrated by van den Boogaard et $\mathrm{al}^{23}$ and Zhang et al. ${ }^{16}$
In our study, we did not find any statistical significance correlating CRP levels and delirium, and the explanation could be that we had a relatively small sample of patients. We also did not manage to measure procalcitonine levels, as we based our results on everyday routine blood tests only; this is something that limited the spectrum of findings in our study.

Unlike other studies, we did not find albumin levels to be statistically significant, in contrast to what has been demonstrated in Rudolph et al's ${ }^{24}$ study. The most probable answer for this might be the fact that we had a rather small number of patients with delirium who were documented and analyzed.

\section{Study limitations}

Our study had some clinical limitations. It was conducted in a single hospital, and only a small sample of population (179 patients) was investigated. The study was also based on our own resources with no further funding.

As a result, we used the everyday, routine blood tests in order to carry out our research and extract our conclusions without measuring any other specified biomarkers.

The need for research on this topic on a larger scale is obvious. Such research should be based on multicenter data collection. Prospective studies with a much greater sample size would be needed in order to determine the causality of certain risk factors for delirium.

\section{Conclusion}

We feel confident in declaring that, for the most part, patients who are older, have an extended intubation period, and a prolonged ICU and in-hospital LOS are much more vulnerable to developing delirium in the ICU post cardiac surgery.

In terms of identifying certain biomarkers for early recognition of delirium, the increased NL ratio on the second postoperative day seemed to be a contributing factor to developing delirium in the ICU.

Moreover, changes in the postoperative hematocrit, $\mathrm{Hb}$, and platelets are correlated with delirium development as they affect patients' general condition of health.

In addition, increased perioperative urea, creatinine, and sodium levels in those cases which highlighted the imminent appearance of delirium reflect the necessity to focus on these patients before they deteriorate and develop delirium, resulting in a prolonged hospitalization in the CICU.

Our intention to build a diagnostic tool for delirium (which would consist of RASS and CAM-ICU score, accompanied by certain biomarker results) in order to discern in a timely manner which of the cardiac patients are prone to developing delirium is at its initial steps yet, and still many 
initiatives need to be taken. These initiatives would strengthen our efforts to implement measures for the optimal nursing and medical interventions in order to prevent delirium development in cardiac surgical patients.

\section{Disclosure}

The authors report no conflicts of interest in this work.

\section{References}

1. American Psychiatric Association. Diagnostic and Statistical Manual of Mental Disorders (DSM-5). 5th ed. Washington, DC: American Psychiatric Publishing; 2013.

2. Norkiene I, Ringaitiene D, Misiuriene I, et al. Incidence and precipitating factors of delirium after coronary artery bypass grafting. Scand Cardiovasc J. 2007;41(3):180-185.

3. Meagher D. Motor subtypes of delirium: past, present and future. Int Rev Psychiatry. 2009;21(1):59-73.

4. Stransky M, Schmidt C, Ganslmeier P, et al. Hypoactive delirium after cardiac surgery as an independent risk factor for prolonged mechanical ventilation. J Cardiothorac Vasc Anesth. 2011;25(6):968-974.

5. Arend E, Christensen M. Delirium in the intensive care unit: a review. Nurs Crit Care. 2009;14(3):145-154.

6. Adamis D, Dimitriou C, Anifantaki S, et al. Validation of the Greek version of confusion assessment method for the intensive care unit (CAM-ICU). Intensive Crit Care Nurs. 2012;28(6):337-343.

7. Sessler CN, Gosnell MS, Grap MJ, et al. The Richmond Agitation - Sedation Scale: validity and reliability in adult intensive care unit patients. Am J Respir Crit Care Med. 2002;166(10):1338-1344.

8. American Psychiatric Association, Practice guideline for the treatment of patients with delirium, 1999.

9. Li HC, Chen YS, Chiu MJ, Fu MC, Huang GH, Chen CC. Delirium, subsyndromal delirium, and cognitive changes in individuals undergoing elective coronary artery bypass graft surgery. $J$ Cardiovasc Nurs. 2015;30(4):340-345.

10. Bakker RC, Osse RJ, Tulen JH, Kappetein AP, Bogers AJ. Preoperative and operative predictors of delirium after cardiac surgery in elderly patients. Eur J Cardiothorac Surg. 2012;41(3):544-549.

11. Weinrebe W, Johannsdottir E, Karaman M, Füsgen I. What does delirium cost? An economic evaluation of hyperactive delirium. Z Gerontol Geriatr. 2016;49(1):52-58.

12. Lee E, Kim J. Cost-benefit analysis of a delirium prevention strategy in the intensive care unit. Nurs Crit Care. Epub October 29, 2014.
13. Franco K, Litaker D, Locala J, Bronson D. The cost of delirium in the surgical patient. Psychosomatics. 2001;42(1):68-73.

14. Androsova G, Krause R, Winterer G, Schneider R. Biomarkers of postoperative delirium and cognitive dysfunction. Front Aging $\mathrm{Neu}$ rosci. 2015;7:112.

15. Rudolph JL, Ramlawi B, Kuchel GA, et al. Chemokines are associated with delirium after cardiac surgery. J Gerontol A Biol Sci Med Sci. 2008;63(2):184-189.

16. Zhang Z, Pan L, Deng H, Ni H, Xu X. Prediction of delirium in critically ill patients with elevated C-reactive protein. J Crit Care. 2014;29(1):88-92.

17. Shadvar K, Baastani F, Mahmoodpoor A, Bilehjani, E. Evaluation of the prevalence and risk factors of delirium in cardiac surgery ICU. J Cardiovasc Thorac Res. 2013;5(4):157-161.

18. Sunbul EA, Sunbul M, Yanartas O, et al. Increased neutrophil/lymphocyte ratio in patients with depression is correlated with the severity of depression and cardiovascular risk factors. Psychiatry Investig. 2016;13(1):121-126.

19. Giakoumidakis K, Fotos NV, Patelarou A, et al. Perioperative neutrophil to lymphocyte ratio as a predictor of poor cardiac surgery patient outcomes. Pragmat Obs Res. 2017;8:9-14.

20. Tan TP, Arekapudi A, Metha J, Prasad A, Venkatraghavan L. Neutrophil-lymphocyte ratio as predictor of mortality and morbidity in cardiovascular surgery: a systematic review. ANZ J Surg. 2015;85(6): 414-419.

21. Egberts A, Mattace-Raso FUS. Increased neutrophillymphocyte ratio in delirium: a pilot study. Clin Interv Aging. 2017;12:1115-1121.

22. Kulaksizoglu B, Kulaksizoglu S. Relationship between neutrophil/ lymphocyte ratio with oxidative stress and psychopathology in patients with schizophrenia. Neuropsychiatr Dis Treat. 2016;12:1999-2005.

23. van den Boogaard M, Kox M, Quinn KL, et al. Biomarkers associated with delirium in critically ill patients and their relation with long-term subjective cognitive dysfunction; indications for different pathways governing delirium in inflamed and noninflamed patients. Crit Care. 2011;15(6):R297.

24. Rudolph JL, Jones RN, Levkoff SE, et al. Derivation and validation of a preoperative prediction rule for delirium after cardiac surgery. Circulation. 2009;119(2):229-236.

25. Tsuruta R, Oda Y. A clinical perspective of sepsis-associated delirium. J Intensive Care. 2016;4:18.

26. Eden BM, Foreman MD. Problems associated with underrecognition of delirium in critical care: a case study. Heart Lung. 1996;25(5):388-400.

27. Steiner LA. Postoperative delirium. Part 1: pathophysiology and risk factors. Eur J Anaesthesiol. 2011;28(9):628-636.

28. Ely EW, Inouye SK, Bernard GR, et al. Delirium in mechanically ventilated patients. JAMA. 2011;286(21):2703-2710.
Pragmatic and Observational Research

\section{Publish your work in this journal}

Pragmatic and Observational Research is an international, peer-reviewed, open access journal that publishes data from studies designed to reflect more closely medical interventions in real-world clinical practice compared with classical randomized controlled trials (RCTs). The manuscript management system is completely online and includes a very quick and fair peer-review

\section{Dovepress}

system. Visit http://www.dovepress.com/testimonials.php to read real quotes from published authors. 